\title{
Repetitive Transient Extraction Using the Optimized SES Entropy Wavelet for Fault Diagnosis of Rotating Machinery
}

\author{
Tingzhong Wang $\mathbb{C D}^{1}{ }^{1}$ Lingli Zhu, ${ }^{1}$ Miaomiao $\mathrm{Fu}^{1}{ }^{1}$ Tingting $\mathrm{Zhu}^{1}$, and Ping $\mathrm{He}^{2}$ \\ ${ }^{1}$ School of Information Technology, Luoyang Normal University, Luoyang 471934, China \\ ${ }^{2}$ HIKVISION, Hangzhou, Zhejiang 310000, China \\ Correspondence should be addressed to Tingzhong Wang; wangtingzhong@lynu.edu.cn
}

Received 6 October 2021; Accepted 23 November 2021; Published 20 December 2021

Academic Editor: Guo-Qing Zhang

Copyright (c) 2021 Tingzhong Wang et al. This is an open access article distributed under the Creative Commons Attribution License, which permits unrestricted use, distribution, and reproduction in any medium, provided the original work is properly cited.

\begin{abstract}
Repetitive transients are usually generated in the monitoring data when a fault occurs on the machinery. As a result, many methods such as kurtogram and optimized Morlet wavelet and kurtosis method are proposed to extract the repetitive transients for fault diagnosis. However, one shortcoming of these methods is that they are constructed based on the index of kurtosis and are sensitive to the impulsive noise, leading to failure in accurately diagnosing the fault of the machinery operating under harsh environment. To address this issue, an optimized SES entropy wavelet method is proposed. In the proposed method, the optimized parameters including bandwidth and central frequency of Morlet wavelets are selected. Then, based on the wavelet coefficients decomposed using the optimized Morlet wavelet, the SES entropy is calculated to select the scales of wavelet coefficients. Finally, the repetitive transients are reconstructed based on the denoising wavelet coefficients of the selected scales. One simulation case and vibration data collected from the experimental setup are used to verify the effectiveness of the proposed method. The simulated and experimental analyses showed that the signal-to-noise ratio (SNR) of the proposed method has the largest value. Specifically, the SNR in the experimental analysis of the proposed method is 0.6 , while that of the other three methods is 0.043 , 0.0065 , and 0.0045 , respectively. Therefore, the result shows that the proposed method is superior to the traditional methods for repetitive transient extraction from the vibration data suffered from impulsive noise.
\end{abstract}

\section{Introduction}

Fault diagnosis plays a vital role in ensuring long-term safe running of rotating machinery for avoiding huge economic loss and casualties. Thus, many fault diagnosis methods based on the collected monitoring signals such as sound [1], infrared images [2], and current [3] are carried out. Of all the different types of monitoring signal, vibration signal contains abundant information of machinery health conditions and thus is the most analyzed signal, and many signal processing based methods have been proposed to process vibration signals for fault diagnosis $[4,5]$. The abundant information refers to the repetitive transients generated periodically when faults occur on the rotating machinery [6]. By analyzing the frequency of these transients, it is easy to know which part has faults or dynamic changes [7].
Although vibration signals containing repetitive transients can be used for diagnosing the fault, machinery usually operates under harsh environment and the transients are submerged in noise. As a result, it is difficult to extract useful information from vibration signals and inaccurate results probably obtained with ineffective methods. To solve this problem, Qiao et al. [8-11] applied stochastic resonance to fault diagnosis, making use of the enhancement of noise to periodicity signals generated due to the faults. Multistable stochastic resonance [12] can also be found for fault detection. However, selecting the suitable optimal parameters of stochastic resonance is not an easy thing; although the stochastic resonance can exhibit the energy of noise for transient extraction, these signal processing-based methods cannot be widely used in real applications. Moreover, blind deconvolution technique [13] and minimax concave 
regularized sparse representation [14] are also used for extracting repetitive transients for fault diagnosis, but they are not robust to heavy noise.

Time-frequency signal processing techniques including wavelet transform and empirical mode decomposition are also commonly used to process vibration signals for extracting useful fault information. The empirical mode decomposition and its variants [15] can be found to extract repetitive transients in fault diagnosis of rotating machines. These methods decompose vibration signals into different models of intrinsic model functions and then the signals can be classified into nonstationary and nonlinear components for analysis. However, it should be noted that these methods have many problems such as endpoint effects, modal aliasing, and lacking mathematical theory, which limit the use of these methods. Wavelet transform is another commonly seen time-frequency analysis method and is performed by decomposing signals into different scales of frequency bands. Continuously, the frequency bands of repetitive transients can be selected by choosing scales for the reconstruction of repetitive transients. Zhang et al. [16] achieved the bearing fault diagnosis based on multiple Q-factor Gabor wavelets. Mishra et al. [17] used envelope analysis and wavelet denoising to diagnose rolling element bearing fault under slow-speed operation. In recent years, more improved wavelet-based diagnosis methods including M-band flexible wavelet transform [18], empirical wavelet transform $[19,20]$, tunable Q-factor wavelet transform [21], and dynamic Bayesian wavelet transform [22] are proposed. But there exist some common issues for these wavelet-transform-based methods. First, it is difficult to choose a suitable wavelet function that should be similar to the transients. A random selection of wavelet function may lead to the wrong representation of transients and then no useful information is probably obtained. Second, there are no effective theories to guide the selection of wavelet scales. The wavelet scales representing the frequency of repetitive transients should be selected, but which scales should be selected should be guided with an effective index.

Kurtosis is sensitive to impulsive signals and exhibits large values when there are impulsive signals [23]. Based on these concepts, many useful kurtosis-based diagnosis methods have been proposed. Antoni [24] constructed a kurtogram method, which uses short-time Fourier transform to decompose signals into different frequency bands and select the optimal frequency band based on the index of kurtosis. Wang [25] proposed an enhanced kurtogram method for fault diagnosis of rolling element bearings based on the binary wavelet packet transform. Chen [26] constructed an improved fast spatialspectral ensemble kurtosis kurtogram and applied it to mechanical signature analysis of short duration data from rotating machinery. By combining minimum entropy deconvolution and spectral kurtosis, a new l0-norm embedded MED method was proposed for roller element bearing fault diagnosis at the early stage of damage [27]. A sub-band averaging kurtogram was proposed by incorporating with dual-tree complex wavelet packet transform [28]. Adaptive correlated kurtogram was constructed based on scale-space representation and empirical wavelet transform [29]. Qin [30] proposed an optimized Morlet wavelet and kurtosis method, in which the kurtosis is used to select the optimal scales. Although kurtosis is sensitive to repetitive transients, it is also more sensitive to the single impulsive noise. The single impulsive noise is widely seen because the machinery usually operates under harsh environment, and disturbances are inevitable. The disturbance for short time can lead to impulsive noise. As a result, these kurtosis methods probably fail to detect fault from the data containing impulsive noise. Infogram was proposed to overcome this issue by calculating square envelope spectrum entropy, but this method is not robust to heavy noise and there are usually several frequency bands appearing with large values [30]. Afterward, the square envelope spectrum entropy has attracted much attention and many variants of infogram have been constructed [31-34].

To effectively extract repetitive transients from signals suffered from heavy noise including the Gaussian white noise and the impulsive noise, a new fault diagnosis method is proposed based on the optimized Morlet wavelet and the square envelope spectrum entropy. In the proposed method, the Morlet wavelet function which is similar to the impulsive signals is used. The parameters of Morlet including bandwidth and the central wavelet frequency are optimized according to the Shannon entropy of wavelet coefficients. Based on the wavelet transform using the optimal Morlet wavelet function, square envelope spectrum entropy is calculated for guiding the selection of wavelet scales. Finally, the repetitive transients can be reconstructed based on the selected wavelet coefficients after denoising. The effectiveness of the proposed method is demonstrated using simulation signal and experimental signal, respectively. The proposed method can be used for the fault diagnosis of machinery suffered from both heavy Gaussian white noise and impulsive noise. Impulsive noise is widely seen in the collected data due to random disturbance, and traditional methods such as kurtogram cannot be used to process these data effectively. Thus, the proposed method is suitable for a wide range of applications in real engineering.

Based on the previously mentioned statements, the contributions of this paper are summarized as follows:

(1) An optimized SES entropy wavelet is proposed to extract the repetitive transients for fault diagnosis, and the proposed method presents good performance even when impulsive noise is mixed in the diagnosed signal

(2) The Morlet wavelet function which is similar to impulsive signals is used and its parameters are optimized and selected by calculating the Shannon entropy of wavelet coefficients

(3) Square envelope spectrum entropy is introduced for guiding the selection of wavelet scales

The rest of this paper is organized as follows. Section 2 is devoted to introduce the basic theory of wavelet transforms. In Section 3, the detailed procedure of the proposed method is present. In Section 4, the proposed method is demonstrated based on simulated and experimental signals, respectively. Finally, the conclusions are drawn in Section 5. 


\section{The Theory of Wavelet Transform}

Wavelet transform is widely used to analyze the signals in the time-frequency domain and is efficient for capturing the time of occurrence of a particular frequency. Accordingly, this method is robust for analyzing nonstationary signals and thus used in the proposed method. To help a better understanding of the proposed method, the theory of wavelet transform is introduced in detail.

Wavelet can be considered as a short-duration wave growing and decaying over a limited period. The wavelet denoted using $\psi(t)$ satisfies the following two conditions:

$$
\begin{aligned}
\int_{-\infty}^{+\infty} \psi(t) \mathrm{d} t & =0, \\
\int_{-\infty}^{+\infty}|\psi(t)|^{2} \mathrm{~d} t & =1 .
\end{aligned}
$$

By scaling and shifting $\psi(t)$ through $a$ and $b$, respectively, a set of basis functions $\psi_{a, b}(t)$ can be obtained according to

$$
\psi_{a, b}(t)=\frac{1}{\sqrt{a}} \psi\left(\frac{t-b}{a}\right) \mathrm{d} t ; \quad a>0,(t, a, b) \in R .
$$

Wavelet transform is a powerful mathematic tool for obtaining a representation, decomposition, and reconstruction of signals. Specifically, wavelet transform is performed by decomposing the studied signals into a combination of time-shifted and dilated or compressed local basis functions according to

$$
\mathrm{WT}(a, b)=\int_{-\infty}^{+\infty} x(t) \psi_{a, b}^{*}(t) \mathrm{d} t,
$$

where $x(t)$ denotes the studied signal $b$. The asterisk represents the computation of complex conjugate, and thus, wavelet transform can be considered as a convolution of the signal $x(t)$ with a window $\psi_{a, b}(t)$ that is dilated by $a$ and shifted in time by $b$.

As a result, each wavelet can be located at a different position around $b$ along the time axis. Furthermore, with the help of $a$, wavelets of different frequencies can be obtained. $a$ with large values indicating that the frequency of wavelet of frequency is low, while $a$ with small values correspond to high frequency.

\section{The Proposed Method}

In this section, a repetitive transient extraction method based on an optimized SES entropy wavelet is constructed and introduced for machinery fault diagnosis. The flowchart of the proposed method is graphically shown in Figure 1, and its detailed procedures are described as follows:

(1) First, the monitoring data are collected from machinery and used for fault diagnosis, and some parameters of the Morlet wavelet including $f_{b}$ and $f_{c}$ are initialized. $f_{b}$ and $f_{c}$ represent the bandwidth and the central wavelet frequency, respectively.

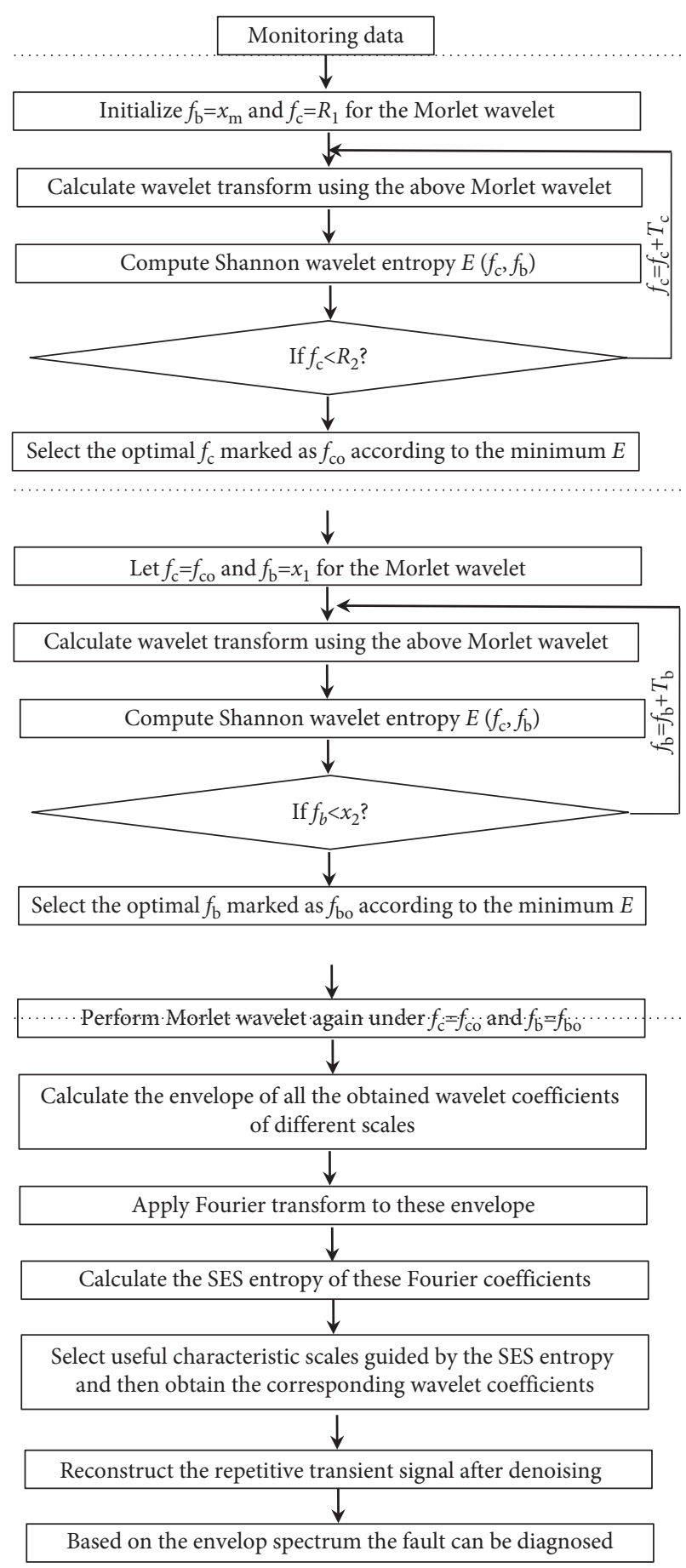

FIgURE 1: The flowchart of the proposed fault diagnosis method.

The Morlet wavelet which is similar to the impulsive signal generated due to faults is used in wavelet transform. The Morlet transform is shown as

$$
\psi(t)=\frac{1}{\sqrt{\pi f_{b}}} \exp \left(\frac{-t^{2}}{f_{b}}\right) \cos \left(2 \pi f_{c} t\right) .
$$

It can be found that the oscillation attenuation depends greatly on $f_{b}$, specifically, the increase of $f_{b}$ enhances the oscillating of the Morlet wavelet, $f_{c}$ 
controls the oscillatory frequency, and the increase of $f_{c}$ leads to the decrease of the frequency resolution of the Morlet wavelet. To find the optimal $f_{c}$, $f_{b}$ should be set as a large value. Therefore, $f_{b}$ is set to be 30 , while $f_{c}$ is set as 0.2 . $f_{c}$ will be iterated from 0.2 to 1 with a step of 0.1 , while $f_{b}$ remain unchanged.

(2) Wavelet transform is calculated based on the Morlet wavelet according to equation (4), so the wavelet coefficients of different scales can be obtained.

(3) Wavelet Shannon entropy is computed using

$$
E\left(f_{c}, f_{b}\right)=-\sum_{i=1}^{K} p_{i} \log p_{i}
$$

where $p_{i}$ is the distribution sequence calculated based on wavelet coefficients according to

$$
p_{i}\left(f_{c}, f_{b}\right)=\frac{W\left(a_{i}, t\right)}{\sum_{j=1}^{k} W\left(a_{j}, t\right)}
$$

(4) If $f_{c}$ does not exceed R2, iterate $f_{c}$ with an increase in $T_{c}$ and then compute the new wavelet Shannon entropy according to these above steps.

(5) The iteration stops until $f_{c}$ reaches $\mathrm{R} 2$ and selects the optimal $f_{c}$ which is marked as $f_{c o} . f_{c}$ is selected as $f_{c o}$ when $E\left(f_{c}, f_{b}\right)$ reach the minimum.

(6) The similar procedure will be performed to find the optimal $f_{b}$ and set $f_{c}$ as $f_{c o}$.

During this process, $f_{b}$ is varied from $\left[x_{1}, x_{2}\right]$, where $x_{1}$ and $x_{2}$ is set to be 0 and 40 , respectively. The optimal $f_{b}$ is marked as $f_{b o}$ and selected when $E\left(f_{c o}, f_{b}\right)$ reach the minimum.

(7) The Morlet wavelet transform is performed again on the monitoring data, where $f_{c}$ and $f_{b}$ is set as $f_{c o}$ and $f_{b o}$, respectively. As a result, wavelet coefficients of different scales can be obtained and denoted using $\left|W\left(a_{i}, t\right)\right|$.

(8) The square envelope of these coefficients can be calculated using

$$
\omega\left(a_{i}, t\right)=\left|W\left(a_{i}, t\right)\right|^{2} .
$$

(9) Based on the discrete Fourier transform, the square envelope spectrum (SES) can be calculated using

$$
\omega \widetilde{\left(a_{i}, \alpha\right)}=\sum_{n=0}^{L-1} W\left(a_{i}, n\right) e^{-j 2 \pi \alpha n / F_{s}}
$$

where $\alpha$ denotes the cyclic frequency and $F_{s}$ is the sampling frequency.

(10) The SES negentropy of these Fourier coefficients is calculated according to the following equation:

$$
\operatorname{SES}\left(a_{i}\right)=\left\langle\frac{\omega \widetilde{\left(a_{i}, \alpha\right)^{2}}}{\left\langle\omega \widetilde{\left(a_{i}, \alpha\right)^{2}}\right\rangle} \ln \left(\frac{\omega \widetilde{\left(a_{i}, \alpha\right)^{2}}}{\left\langle\omega \widetilde{\left(a_{i}, \alpha\right)^{2}}\right\rangle}\right)\right\rangle \text {. }
$$

(11) The characteristic scales whose SES negentropy satisfies the following conditions are selected as the useful scales for reconstructing repetitive transients:

$$
\operatorname{SES}\left(a_{i}\right)>T * \max (\mathrm{SES}) \text {, }
$$

where $T$ is a threshold value and set to be 0.75 . In this way, the wavelet coefficients with larger SES negentropy can be selected and they mostly contain repetitive transients.

(12) The wavelet coefficients of the selected characteristics are denoised using soft thresholding to enhance the ability of transient extraction according to

$$
W^{\prime}\left(a_{i}, n\right)= \begin{cases}\operatorname{sgn}\left(W\left(a_{i}, n\right)\right)\left(\left|W\left(a_{i}, n\right)\right|-\theta\right), & \left|W\left(a_{i}, n\right)\right|>\theta, \\ 0, & \left|W\left(a_{i}, n\right)\right| \leq \theta,\end{cases}
$$

where $\theta$ is a threshold value.

(13) The repetitive transients can be extracted by reconstructing the denoised wavelet coefficients using

$$
s(n)=\frac{1}{C_{1 \psi}} \sum_{a} W^{\prime}(a, n) a^{-3 / 2}
$$

where $C_{1 \psi}=\int_{-\infty}^{+\infty} \widehat{\psi^{*}}(w) /|w| \mathrm{d} w$.
In a continued manner, the reconstructed repetitive transients can be used for fault diagnosis by calculating the Hilbert envelop spectrum of these transients.

\section{Simulation and Experimental Demonstrations}

4.1. Transient Extraction for Simulated Signal of Bearing Faults. In order to demonstrate the effectiveness of the proposed method for repetitive transient extraction, the simulated signals of the fault bearing are considered in this case study. The signal is simulated using the following model: 


$$
x(t)=\sum_{m}^{M-1}\left[A_{m} \exp \left(-\beta_{w}\left(t-m / f_{o}\right)\right) \sin \left(2 \pi f_{r e}\left(t-m / f_{o}\right)\right) u\left(t-m / f_{o}\right)\right]+I(t)+N(t),
$$

where $A_{m}$ represents the amplitude value of the simulated signal. $A_{m}$ is randomly selected and varies from 0.6 to 1.6. $u(t)$ denotes a unit step function, $\beta_{w}$ denotes structure damping ratio, and $\beta_{w}=900$. The resonance frequency $f_{\text {re }}$ and the sampling frequency $f_{s}$ are $3000 \mathrm{~Hz}$ and $12 \mathrm{kHz}$, respectively. The total sampling time is $0.5 \mathrm{~s}$. Moreover, $m$ denotes the number of impulses that periodically appear in the simulated signal. $M$ is the total number of the simulated impulses and is set to 200. $f_{o}$ is the fault characteristic frequency and set to $50 \mathrm{~Hz}$ in this simulation.

In addition, to simulate the impulsive noise due to the harsh environment, $I(t)$ is also added to the simulated signal, and its amplitude, resonance frequency, and bandwidth is $8 \mathrm{~g}, 5 \mathrm{kHz}$, and $800 \mathrm{~Hz}$, respectively. $N(t)$ denotes the additive Gaussian white noise with zero mean and satisfies the following equation:

$$
\left\{\begin{array}{l}
\langle N(t)\rangle=0, \\
\langle N(t), N(t+\tau)\rangle=2 D \delta(\tau),
\end{array}\right.
$$

where $D$ is the intensity of the Gaussian white noise and is selected as 0.125 .

The time domain of waveforms of the simulated signal is shown in Figure 2. From Figure 2(a), it can be found that transients are generated repetitively with a periodicity of $1 / f_{o} \mathrm{~s}$. Unlike the repetitive transients, the impulsive noise appears with a large amplitude. This noise is commonly seen in the real monitoring data due to disturbance from the operational environment. In addition to the impulsive noise, Gaussian white noise is added to the signal shown in Figure 2(a) and the repetitive transients are seriously deteriorated in the signal shown in Figure 2(b). Obviously, it is difficult to extract the repetitive transients for fault diagnosis from the signal using traditional signal processing based fault diagnosis methods such as kurtogram. The proposed method is applied to processing this signal, and the time-domain waveform of the extracted repetitive transients is shown in Figure 3(a). It can be seen that the repetitive transients can be successfully extracted using the proposed method by removing both the Gaussian white noise and the impulsive noise. Then, the Hilbert envelope spectrum of the signal shown in Figure 3(a) is calculated and presented in Figure 3(b). The amplitude of fault frequency and its harmonic frequency such as the second, third, and fourth are high and clearly seen. It can be inferred that a fault occurs on the monitoring bearing, which agrees with the simulated data. Therefore, the proposed method is effective for repetitive transient extraction for fault diagnosis.

For comparison, the traditional methods including kurtogram, infogram, and the optimized Morlet wavelet and kurtosis method are used to process this simulated signal. Kurtogram is a widely used method to extract repetitive transients for fault diagnosis, so this method is considered for comparison. The result of the kurtogram is shown in Figure 4, and it can be found that the large value appears at the center frequency $\left(f_{\mathrm{c}}\right)$ of $4968.75 \mathrm{~Hz}$ and at the level of 7 , which can be detected as the optimal frequency band and marked using a red color line. Consequently, the signal at this frequency band is filtered for extracting the repetitive transients. The extracted repetitive transients using kurtogram are shown in Figure 5(b). Obviously, the impulsive noise is contained in the repetitive transients, indicating the kurtogram cannot be used for repetitive transient extraction when there exists heavy noise in the collected signal. The envelop spectrum of the signal shown in Figure 5(b) is presented in Figure 5(c). There is no useful information presented in the spectrum graph and the bearing fault cannot be successfully detected. Kurtogram is constructed by calculating the kurtosis index in different frequency bands decomposed using a short Fourier transform. The kurtosis index is very sensitive to impulsive noise and has a very large value under a sole impulsive noise. As a result, the frequency band of the impulsive noise will be wrongly selected for fault diagnosis.

Infogram is also proposed by Antoni for overcoming the shortcomings of the kurtogram, which is constructed based on Shannon entropy. The results of the infogram are shown in Figure 6. There are several frequency bands with large values because the infogram is not robust to noise. The frequency band with the largest value is at the center frequency of $4500 \mathrm{~Hz}$ and at the second level, which is also marked using a red color line. The extracted repetitive transients are shown in Figure 7(b), and we can find that both the repetitive transients and the impulsive noise are contained in the repetitive transients. As shown in Figure 7(c), although the amplitude of the fault frequency can also be seen, there are also some disturbances in the envelope spectrum due to the impulsive noise. In sum, the infogram cannot be used to remove the impulsive noise because of its coarsely calculated procedure.

Like the kurtogram, the Morlet wavelet and kurtosis method is constructed based on the index of kurtosis and is sensitive to impulsive noise. The time-domain waveform of the extracted repetitive transients using the Morlet wavelet and kurtosis method is shown in Figure 8(a), in which the impulsive noise rather than the periodically appeared impulsive is clearly seen. As a result, the amplitude at the fault frequency has a small value and there are just low-frequency components in the envelop spectrum, as shown in Figure $8(\mathrm{~b})$. It is because the scale of the signal containing impulsive noise has the largest kurtosis and thus is wrongly selected as the repetitive transients in the comparison method.

For quantitative advantages of the proposed method, the SNR is used for the envelope spectrum of the repetitive transients. The expression of SNR is generally written as 


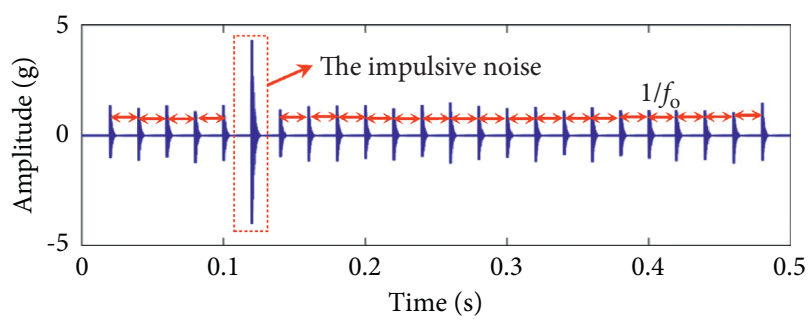

(a)

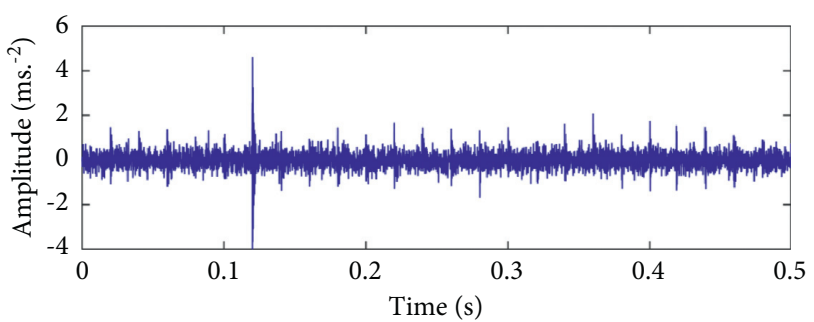

(b)

FIgURE 2: The time-domain waveforms of the simulated signal including (a) the repetitive transients with the impulsive noise and (b) the signal added with Gaussian white noise.

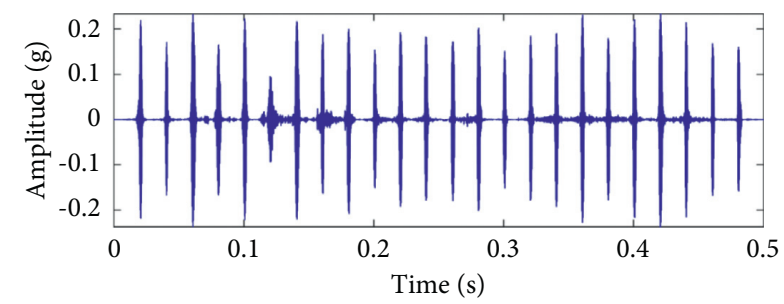

(a)

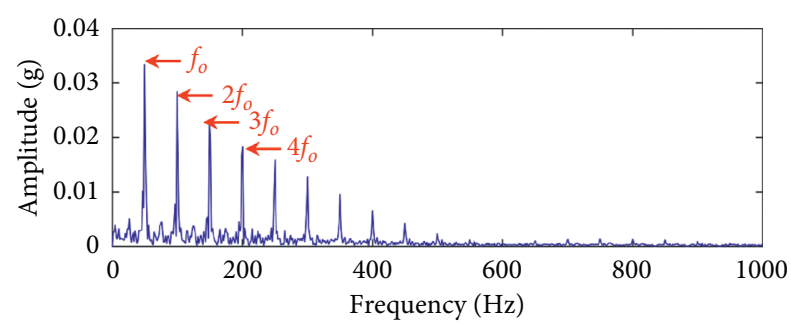

(b)

Figure 3: The result of the proposed method: (a) the extracted repetitive transients and (b) the Hilbert envelop spectrum of (a).

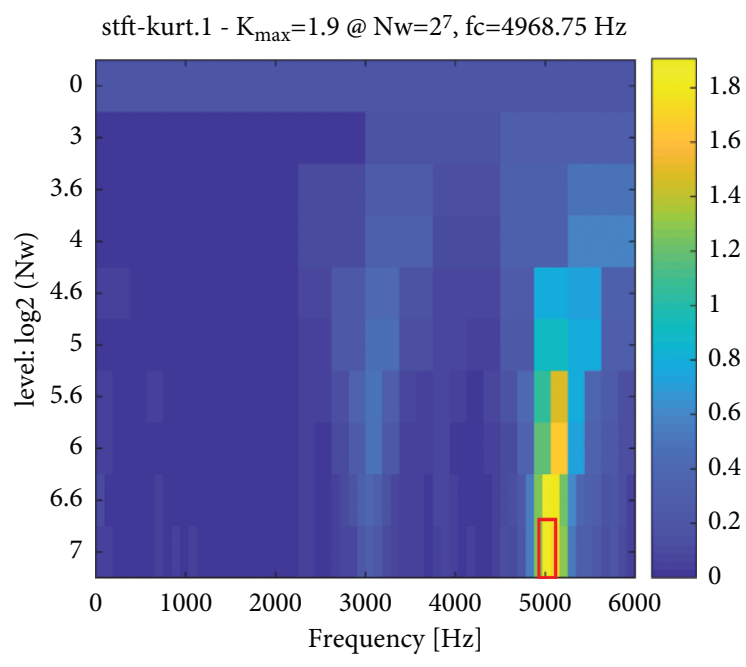

FIgURE 4: The result of the kurtogram.

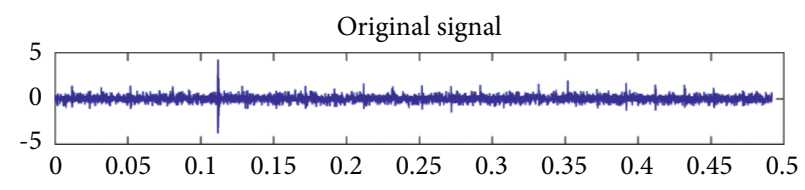

(a)

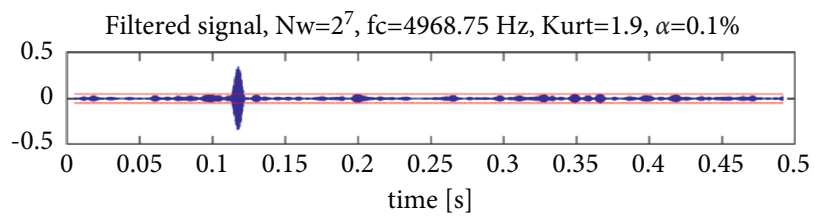

(b)

Figure 5: Continued. 


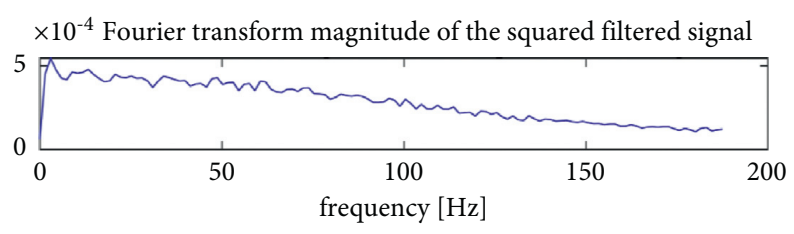

(c)

Figure 5: (a) The signal after prewhitening, (b) the extracted repetitive transients using kurtogram, and (c) the Hilbert envelop spectrum of (b).

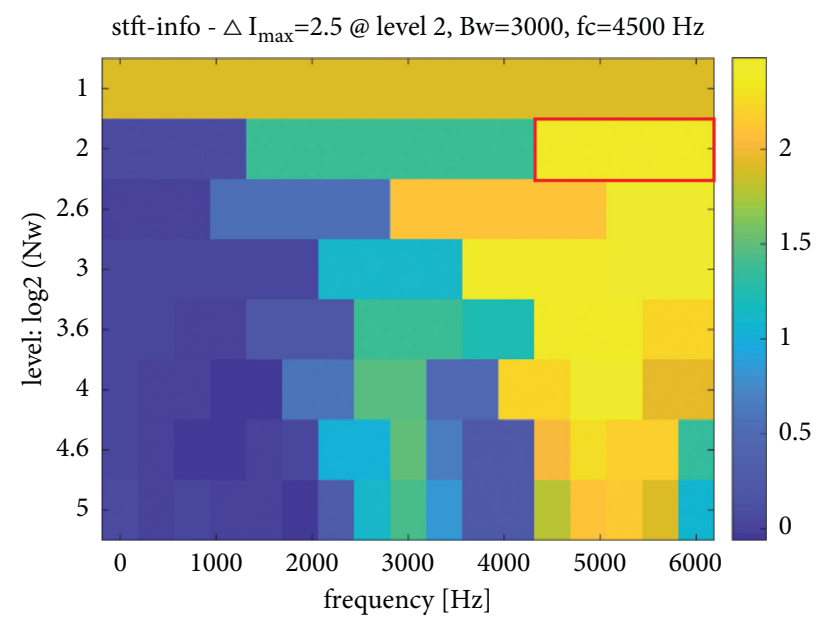

FIgURE 6: The result of infogram.

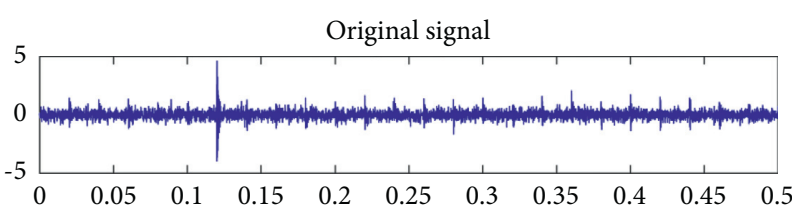

(a)

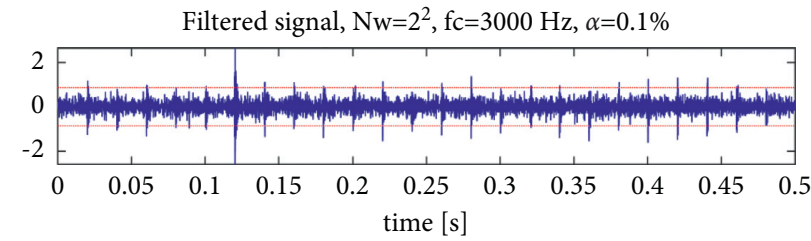

(b)

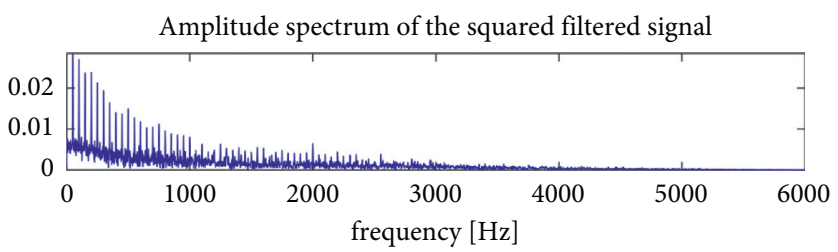

(c)

Figure 7: (a) The signal after prewhitening, (b) the extracted repetitive transients using infogram, and (c) the Hilbert envelop spectrum of (b).

$$
\mathrm{SNR}=10 \log _{10}\left(\frac{\sum_{i}^{N} A_{i}}{E-\sum_{i}^{N} A_{i}}\right)
$$

where $i(0,1, \ldots N)$ denotes the order of the harmonics and $N$ is specified as $4 . A_{i}$ represents the amplitude of the extracted $i$ th harmonic and $E$ is the sum of the amplitude of envelope spectrums. The SNR of the proposed method, infogram, kurtogram, and the Morlet wavelet and kurtosis method can be calculated as $0.135,0.092,0.011$, and
0.0124 , respectively, which verify the effectiveness of the proposed method.

4.2. Transient Extraction for Real Signal Collected from a Fault Bearing. In this section, real signals collected from a fault bearing are used to verify the effectiveness of the proposed method. The data are provided by the bearing data center of Case Western Reserve University [35] and is commonly used for the demonstration of fault diagnosis methods. The 


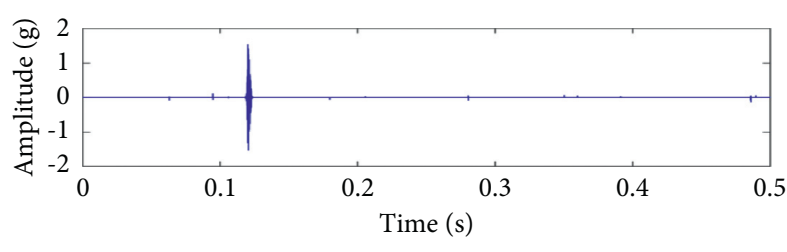

(a)

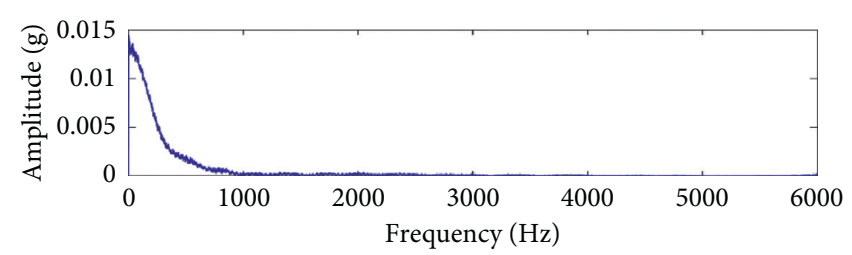

(b)

FIgURE 8: The extraction result of the Morlet wavelet and kurtosis method: (a) the extracted repetitive transients and (b) the Hilbert envelop spectrum of (a).

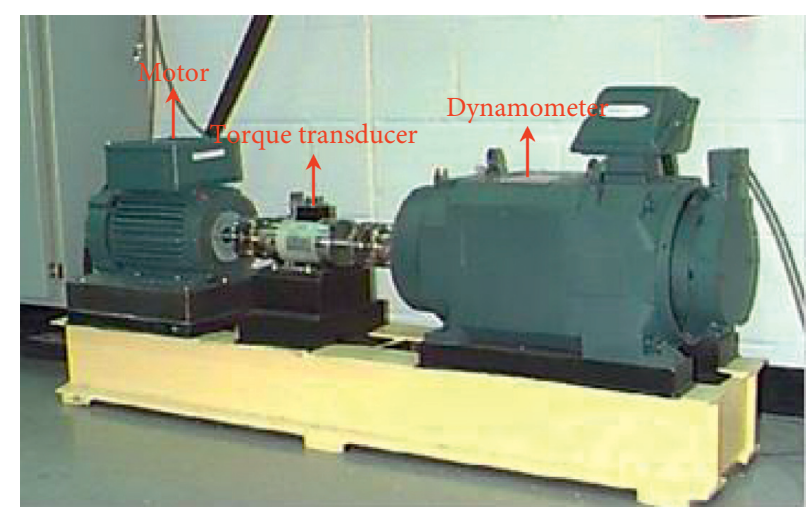

FIGURE 9: The experimental setup.

TABLE 1: The structural parameters of the bearings used in the experiment.

\begin{tabular}{lcccc}
\hline Inner diameter $(\mathrm{mm})$ & Outer diameter $(\mathrm{mm})$ & Pitch diameter $(\mathrm{mm})$ & Contact angle $\left({ }^{\circ}\right)$ & Number of balls \\
\hline 25.001 & 51.999 & 39.040 & 0 & 9 \\
\hline
\end{tabular}

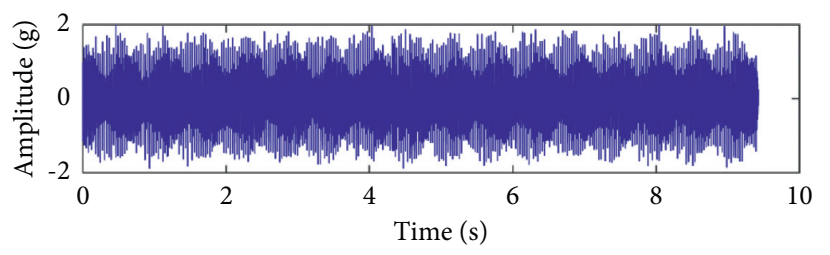

(a)

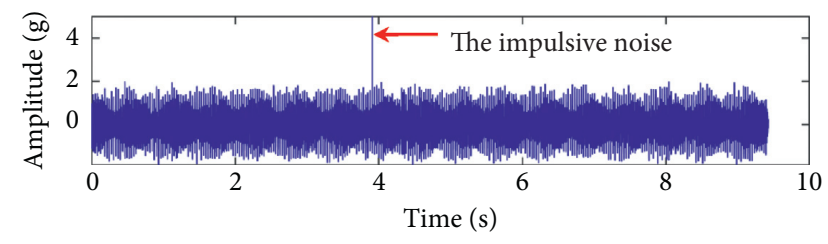

(b)

Figure 10: (a) The collected vibration data and (b) the data added with the impulsive noise.

experimental setup is shown in Figure 9, which consists of a $2 \mathrm{HP}$ motor (left), a torque transducer (center), and a dynamometer (right). The bearing of 6205-2Rs JEM SKF is used for testing, which is installed under the motor shaft. Some structural parameters of the bearing are presented and listed in Table 1. An accelerometer sensor, which is attached to the housing with magnetic bases, is used to collect vibration signals with a sampling of $12 \mathrm{kHz}$.

The vibration signal of fault outer race was collected and processed using the proposed method. The fault is a single-point fault that is produced using electro-discharge machining and the fault diameter is 7 mils ( $1 \mathrm{mil}=0.001$ inches $)$. The rotation speed of the motor is $1797 \mathrm{r} / \mathrm{min}$, and the corresponding outer fault frequency is $91.4 \mathrm{~Hz}$. The time-domain waveform of the vibration signal is shown in Figure 10(a), and it can be found that the repetitive transients are submerged in the noise. Moreover, an impulsive noise whose amplitude value is 5 is introduced into the signal and the corresponding waveform is shown in Figure 10(b).

The proposed method is applied to extract repetitive transients from this signal for fault diagnosis. The timedomain waveform of the repetitive transients is shown in Figure 11(a). It can be found that the simulated impulsive noise is not contained in the signal, indicating that the proposed method is not sensitive to the noise. To further verify the effectiveness of repetitive transients extracting and diagnosis the fault of bearing, the envelope spectrum of the signal is given in Figure 11(b). The amplitude of the outer 


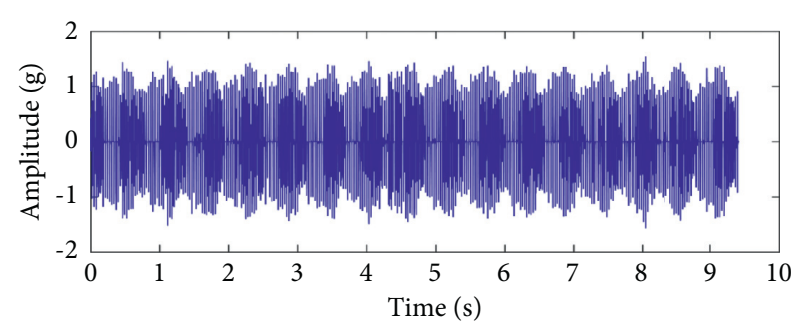

(a)

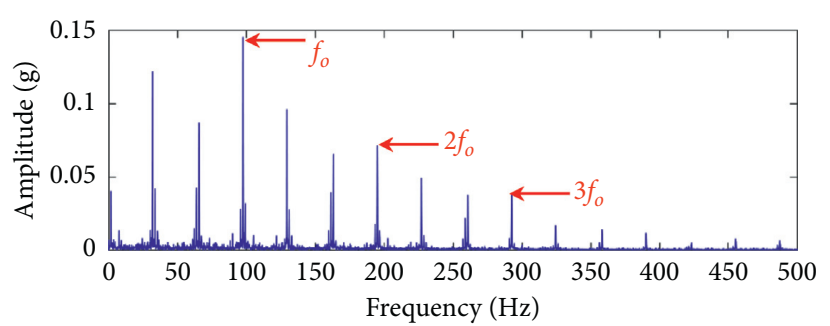

(b)

Figure 11: (a) The extracted repetitive transients using the proposed method and (b) the Hilbert envelop spectrum of (a).

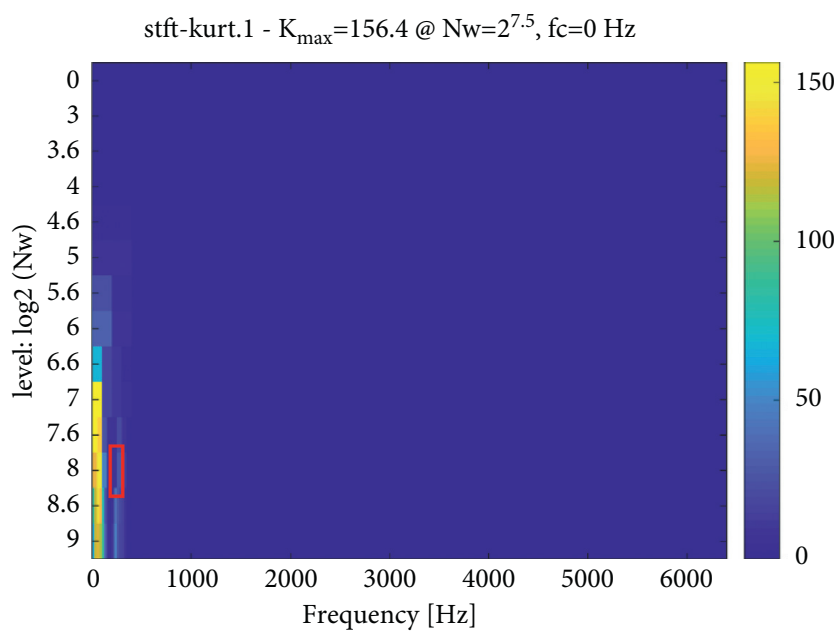

Figure 12: The extraction result of the kurtogram.

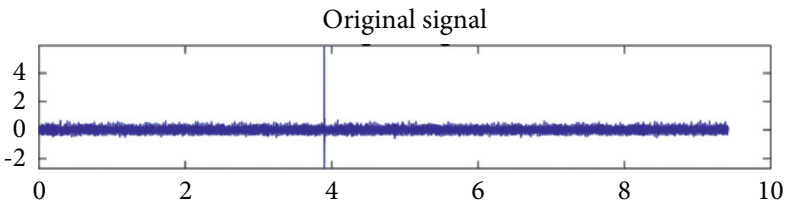

(a)

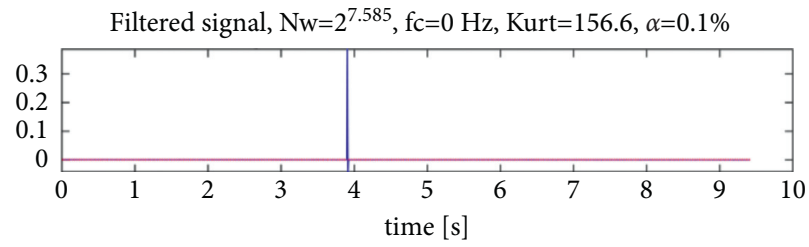

(b)

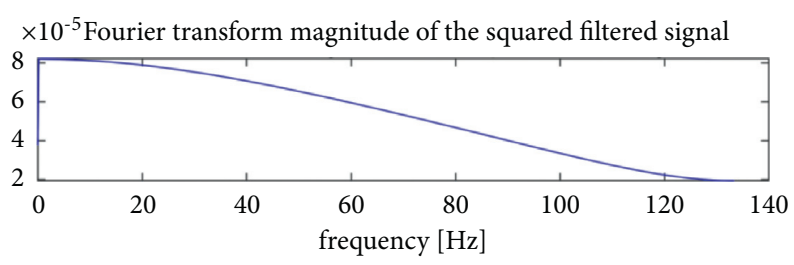

(c)

Figure 13: (a) The data after prewhitening, (b) the extracted repetitive transients using kurtogram, and (c) the Hilbert envelope spectrum of (a).

race fault frequency and its harmonic frequency can be clearly seen. It can be inferred that the bearing suffers from the outer fault, which agrees well with the fact.

The traditional methods are also used for comparison. The results of the kurtogram show that the center frequency of the repetitive transients is $0 \mathrm{~Hz}$ at the $7.5^{\text {th }}$ level (seen in Figure 12), but the extracted signal of the corresponding frequency marked using the red line contains the impulsive noise (seen in Figure 13(b)), and as shown in Figure 13(c), there is no useful information in its Hilbert envelop spectrum. The center frequency at $0 \mathrm{~Hz}$ and $2^{\text {nd }}$ level is selected for repetitive transient extraction using infogram, as shown in Figure 14. The extracted repetitive transients are shown in Figure 15(b), and no useful information can also be found in its Hilbert envelop spectrum, 


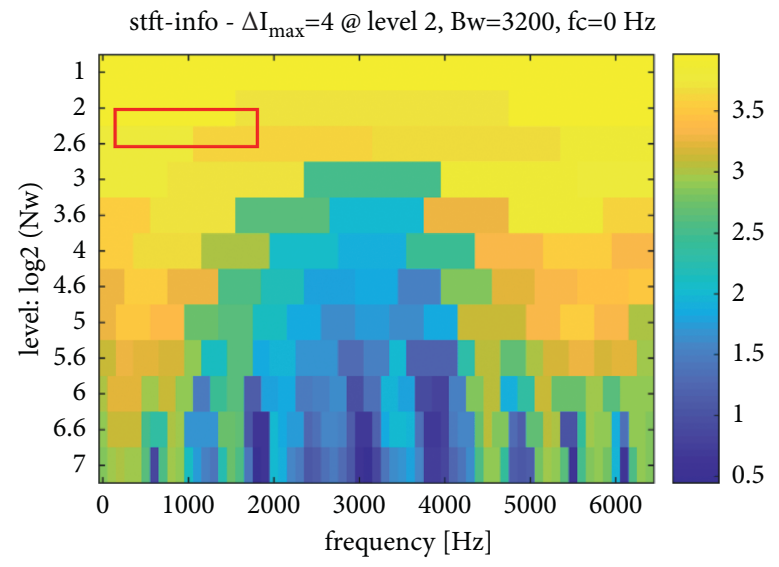

Figure 14: The result of the infogram.

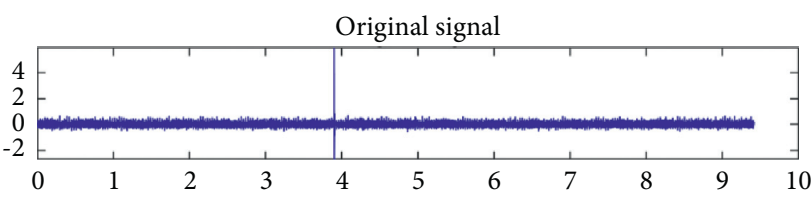

(a)

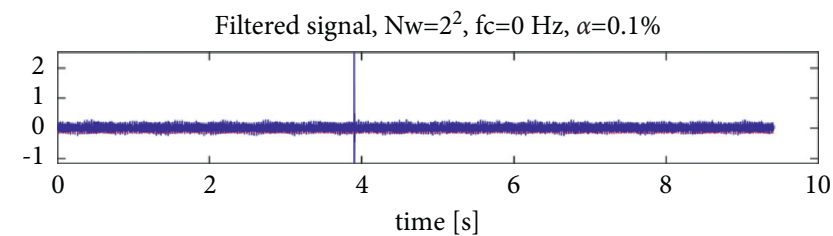

(b)

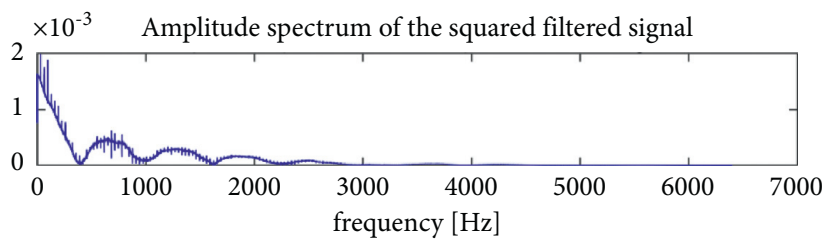

(c)

Figure 15: (a) The data after prewhitening, (b) the extracted repetitive transients using infogram, and (c) the Hilbert envelope spectrum of (a).

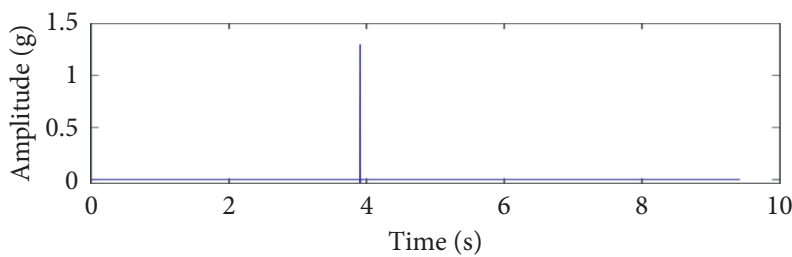

(a)

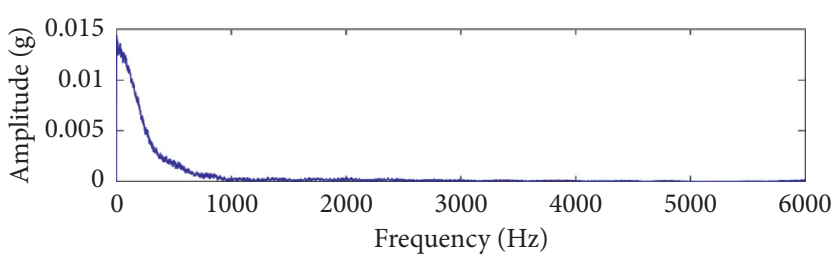

(b)

FIgURE 16: The extraction results of the Morlet wavelet and kurtosis method: (a) the extracted repetitive transients and (b) the Hilbert envelope spectrum of (a).

as shown in Figure 15(c). As shown in Figure 16, a similar result is found, when the optimized Morlet wavelet and kurtosis method is applied to the signal. The SNR of infogram, kurtogram, and the Morlet wavelet and kurtosis method can be calculated as $0.043,0.0065$, and 0.0045 , respectively, while the SNR of the proposed method is 0.6 .

In sum, the proposed method is superior to the traditional methods and is robust to repetitive transient extraction from the signal suffering impulsive noise.

\section{Conclusions}

This paper presents a repetitive transient extraction method based on the optimized SES entropy wavelet for machinery fault diagnosis. In the proposed method, the parameters including bandwidth and central wavelet frequency are optimized by computing wavelet Shannon entropy. The wavelet transform of monitoring signal using the Morlet wavelet under these optimized parameters is performed. 
Next, the square envelope of wavelet coefficients is calculated and then the SES entropy can be obtained. The repetitive transients can be reconstructed using the denoised wavelet coefficients for fault diagnosis. The effectiveness of the proposed method is verified by the simulated and experimental signals. Traditional methods including kurtogram, infogram, and the optimized Morlet wavelet and kurtosis method are used for comparison. The results show that the proposed method is robust to extract repetitive transients submerged in impulsive noise, while traditional methods usually fail to do that because they are sensitive to impulsive noise. Therefore, the proposed method has a significant advantage over traditional methods and is suitable for fault diagnosis of machinery operating under harsh environment. In future, some useful optimal methods [36-38] will be introduced to make the proposed method more robust.

\section{Data Availability}

The data that support the findings of this study are available on request from the corresponding author.

\section{Conflicts of Interest}

The authors declare that they have no conflicts of interest.

\section{Acknowledgments}

This work was supported in part by the Science and Technology Key Project of Henan Province under Grant no. 212102210520.

\section{References}

[1] E. Mollasalehi, D. Wood, and Q. Sun, "Indicative fault diagnosis of wind turbine generator bearings using tower sound and vibration," Energies, vol. 10, no. 11, 2017.

[2] T. Bai, J. Yang, D. Yao, and Y. Wang, "Information fusion of infrared Images and vibration signals for coupling fault diagnosis of rotating machinery," Shock and Vibration, vol. 2021, Article ID 6622041, 2021.

[3] M. Zhang, J. Yang, and Z. Zhang, "Locomotive gear fault diagnosis based on wavelet bispectrum of motor current," Shock and Vibration, vol. 2021, Article ID 5554777, 2021.

[4] F. Miao, R. Zhao, and X. Wang, "A new method of denoising of vibration signal and its application," Shock and Vibration, vol. 2020, Article ID 7587840, 2020.

[5] R. B. Randall and J. Antoni, "Rolling element bearing diagnostics-A tutorial," Mechanical Systems and Signal Processing, vol. 25, no. 2, pp. 485-520, 2011.

[6] J. Antoni and R. B. Randall, "A stochastic model for simulation and diagnostics of rolling element bearings with localized faults," Journal of Vibration and Acoustics, vol. 125, no. 3, pp. 282-289, 2003.

[7] T. Jin, H. Ding, H. Xia, and J. Bao, "Reliability index and Asian barrier option pricing formulas of the uncertain fractional first-hitting time model with Caputo type," Chaos, Solitons \& Fractals, vol. 142, Article ID 110409, 2021.

[8] Z. Qiao, J. Liu, X. Ma, and J. Liu, "Double stochastic resonance induced by varying potential-well depth and width," Journal of the Franklin Institute, vol. 358, no. 3, pp. 2194-2211, 2021.
[9] Z. Qiao and X. Shu, "Coupled neurons with multi-objective optimization benefit incipient fault identification of machinery," Chaos, Solitons \& Fractals, vol. 145, Article ID 110813, 2021.

[10] Z. Qiao, A. Elhattab, X. Shu, and C. He, "A second-order stochastic resonance method enhanced by fractional-order derivative for mechanical fault detection," Nonlinear Dynamics, vol. 106, no. 1, pp. 707-723, 2021.

[11] Z. Qiao, J. Liu, X. Xu, A. Yin, and X. Shu, "Nonlinear resonance decomposition for weak signal detection," Review of Scientific Instruments, vol. 92, no. 10, Article ID 105102, 2021.

[12] H. Cui, Y. Guan, H. Chen, and W. Deng, "A novel advancing signal processing method based on coupled multi-stable stochastic resonance for fault detection," Applied Sciences, vol. 11, no. 12, 2021.

[13] L. He, D. Wang, C. Yi, Q. Zhou, and J. Lin, "Extracting cyclostationarity of repetitive transients from envelope spectrum based on prior-unknown blind deconvolution technique," Signal Processing, vol. 183, Article ID 107997, 2021.

[14] W. Huang, S. Li, X. Fu, C. Zhang, J. Shi, and Z. Zhu, “Transient extraction based on minimax concave regularized sparse representation for gear fault diagnosis," Measurement, vol. 151, Article ID 107273, 2020.

[15] X. Jiang, J. Wang, J. Shi, C. Shen, W. Huang, and Z. Zhu, “A coarse-to-fine decomposing strategy of VMD for extraction of weak repetitive transients in fault diagnosis of rotating machines," Mechanical Systems and Signal Processing, vol. 116, pp. 668-692, 2019.

[16] X. Zhang, Z. Liu, J. Wang, and J. Wang, "Time-frequency analysis for bearing fault diagnosis using multiple Q-factor Gabor wavelets," ISA Transactions, vol. 87, pp. 225-234, 2019.

[17] C. Mishra, A. K. Samantaray, and G. Chakraborty, "Rolling element bearing fault diagnosis under slow speed operation using wavelet de-noising," Measurement, vol. 103, pp. 77-86, 2017.

[18] Y. Qin, Y. Mao, B. Tang, Y. Wang, and H. Chen, "M-band flexible wavelet transform and its application to the fault diagnosis of planetary gear transmission systems," Mechanical Systems and Signal Processing, vol. 134, Article ID 106298, 2019.

[19] W. Teng, X. Ding, H. Cheng, C. Han, Y. Liu, and H. Mu, "Compound faults diagnosis and analysis for a wind turbine gearbox via a novel vibration model and empirical wavelet transform," Renewable Energy, vol. 136, pp. 393-402, 2019.

[20] K. Yu, T. R. Lin, J. Tan, and H. Ma, "An adaptive sensitive frequency band selection method for empirical wavelet transform and its application in bearing fault diagnosis," Measurement, vol. 134, pp. 375-384, 2019.

[21] P. Ma, H. Zhang, W. Fan, and C. Wang, "Early fault diagnosis of bearing based on frequency band extraction and improved tunable Q-factor wavelet transform," Measurement, vol. 137, pp. 189-202, 2019.

[22] D. Wang and K. Tsui, "Dynamic Bayesian wavelet transform: new methodology for extraction of repetitive transients," Mechanical Systems and Signal Processing, vol. 88, pp. 137-144, 2017.

[23] D. Wang, "Spectral L2/L1 norm: a new perspective for spectral kurtosis for characterizing non-stationary signals," Mechanical Systems and Signal Processing, vol. 104, pp. 290-293, 2018.

[24] J. Antoni and R. B. Randall, "The spectral kurtosis: application to the vibratory surveillance and diagnostics of rotating machines," Mechanical Systems and Signal Processing, vol. 20, no. 2, pp. 308-331, 2006.

[25] D. Wang, W. T. Peter, and K. L. Tsui, "An enhanced Kurtogram method for fault diagnosis of rolling element 
bearings," Mechanical Systems and Signal Processing, vol. 35, no. 1-2, pp. 176-199, 2013.

[26] B. Chen, Z. Zhang, Y. Zi, Z. He, and C. Sun, "Detecting of transient vibration signatures using an improved fast spatialspectral ensemble kurtosis kurtogram and its applications to mechanical signature analysis of short duration data from rotating machinery," Mechanical Systems and Signal Processing, vol. 40, no. 1, pp. 1-37, 2013.

[27] X. Jiang, X. Cheng, J. Shi, W. Huang, C. Shen, and Z. Zhu, "A new 10-norm embedded MED method for roller element bearing fault diagnosis at early stage of damage," Measurement, vol. 127, pp. 414-424, 2018.

[28] L. Wang, Z. Liu, H. Cao, and X. Zhang, "Subband averaging kurtogram with dual-tree complex wavelet packet transform for rotating machinery fault diagnosis," Mechanical Systems and Signal Processing, vol. 142, Article ID 106755, 2020.

[29] Z. Liu, S. Yang, Y. Liu, J. Lin, and X. Gu, “Adaptive correlated Kurtogram and its applications in wheelset-bearing system fault diagnosis," Mechanical Systems and Signal Processing, vol. 154, Article ID 107511, 2021.

[30] Y. Qin, J. Xing, and Y. Mao, "Weak transient fault feature extraction based on an optimized Morlet wavelet and kurtosis," Measurement Science and Technology, vol. 27, no. 8, Article ID 085003, 2016.

[31] J. Antoni, "The infogram: entropic evidence of the signature of repetitive transients," Mechanical Systems and Signal Processing, vol. 74, pp. 73-94, 2016.

[32] Z. Feng, H. Ma, and M. J. Zuo, "Spectral negentropy based sidebands and demodulation analysis for planet bearing fault diagnosis," Journal of Sound and Vibration, vol. 410, pp. 124-150, 2017.

[33] A. Moshrefzadeh and A. Fasana, "The Autogram: an effective approach for selecting the optimal demodulation band in rolling element bearings diagnosis," Mechanical Systems and Signal Processing, vol. 105, pp. 294-318, 2018.

[34] Y. Xu, Y. Deng, C. Ma, and K. Zhang, “The Enfigram: a robust method for extracting repetitive transients in rolling bearing fault diagnosis," Mechanical Systems and Signal Processing, vol. 158, Article ID 107779, 2021.

[35] K. A. Loparo and K. A. Loparo, Bearing Data Center, Case Western Reserve University, Cleveland, OH, USA, 2013, http://csegroups.case.edu/bearingdatacenter/pages/ welcomecase-western-reserve-university-bearing-datacenterwebsite.

[36] W. Deng, S. Shang, X. Cai et al., "Quantum differential evolution with cooperative coevolution framework and hybrid mutation strategy for large scale optimization," Knowledge-Based Systems, vol. 224, Article ID 107080, 2021.

[37] W. Deng, J. Xu, H. Zhao, and Y. Song, "A novel gate resource allocation method using improved PSO-based QEA," IEEE Transactions on Intelligent Transportation Systems, 2020.

[38] T. Jin and H. Xia, "Lookback option pricing models based on the uncertain fractional-order differential equation with Caputo type," Journal of Ambient Intelligence and Humanized Computing, pp. 1-14, 2021. 